\title{
Nontuberculous Mycobacterial Infection in Patients with Neurosurgical Hardware: Two Cases and A Review of the Literature
}

\author{
Varun Padmanaban ${ }^{1}$, Rezhan Hussein ${ }^{2}$, Elias Rizk ${ }^{1}$ \\ 1. Neurological Surgery, Penn State Milton S. Hershey Medical Center, Hershey, USA 2. Medicine/Infectious Disease, \\ Penn State Milton S. Hershey Medical Center, Hershey, USA
}

Corresponding author: Varun Padmanaban, vpadmanaban@pennstatehealth.psu.edu

\begin{abstract}
Central nervous system infections with nontuberculous mycobacteria (NTM) are rare but have been increasing in frequency. A small fraction of these infections are related to surgical hardware, with approximately 20 cases reported. Patients typically present with an indolent course but can rapidly deteriorate. We report two novel cases of NTM infection in ventriculoperitoneal shunts, and review the literature on treatment options, challenges and outcomes in these patients. Clinicians should consider NTM when dealing with unusual hardware infections as it is an emerging infectious disease with high potential for morbidity and mortality.
\end{abstract}

Categories: Infectious Disease, Neurosurgery

Keywords: nontuberculous mycobacteria, cns infection, ventriculoperitoneal shunt, review, case report

\section{Introduction}

Nontuberculous mycobacteria (NTM) defined as mycobacterial species other than Mycobacterium tuberculosis complex or Mycobacterium leprae. These organisms are ubiquitous and are found in water, dust and soil. Reports of infection with the organisms have been increasing worldwide, involving the lungs, skin, soft tissue and central nervous system [1]. Central nervous system (CNS) infections with NTM are rare but have high mortality.

Cerebral spinal fluid (CSF) infection from neurosurgical hardware is relatively common with rates ranging from $5 \%$ to $15 \%$ [2]. Most infections develop from colonization of skin flora [2]. We report two cases of NTM infection in neurosurgical patients related to ventriculoperitoneal (VP) shunt hardware, and review the literature, common presentations and current treatment strategies for CNS NTM infections related to neurosurgical hardware.

Received 01/13/2020 Review began 02/20/2020 Review ended 03/20/2020 Published 03/24/2020

(๑) Copyright 2020

Padmanaban et al. This is an open access article distributed under the terms of the Creative Commons Attribution License CC-BY 4.0., which permits unrestricted use, distribution, and reproduction in any medium, provided the original author and source are credited.

\section{Case Presentation}

\section{Patient no. 1}

A 24-year-old man with a history of posterior fossa tumor requiring VP shunting presented with worsening headaches, confusion and weight loss over several months. He had a history of vague pain along the shunt site, chest and abdomen as well as swallowing difficulties over several weeks. The patient had a history of multiple shunt revisions, the most recent of which approximately 10 months prior to our evaluation when he had undergone a proximal shunt revision for an obstructed ventricular catheter.

\section{Patient Course}

A CT of the head and abdomen revealed ventricles stable in size and a pseudocyst around his distal catheter (Figure 1).

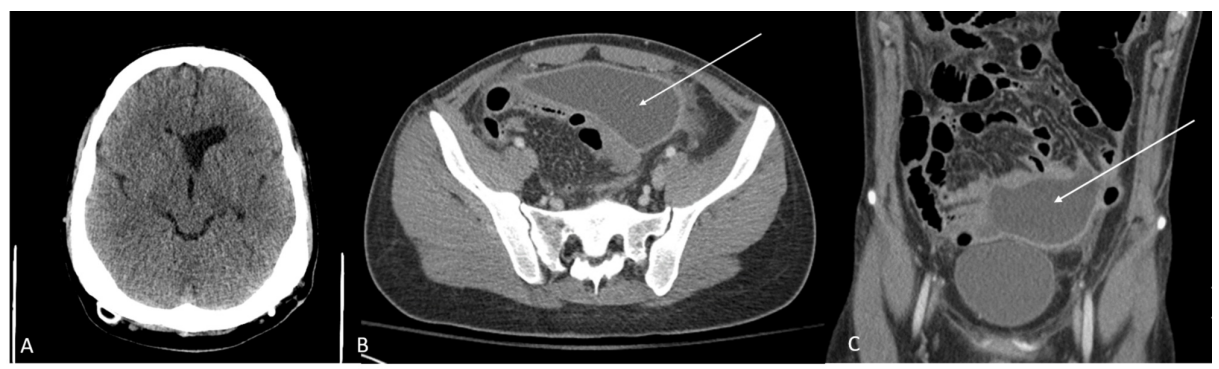




\section{Cureus}

\section{FIGURE 1: CT without contrast of head and abdomen}

CT head without contrast (A) showing stable ventricular size on admission. CT abdomen without contrast showing abdominal pseudocyst (arrow) on axial (B) and coronal (C) sequences.

He was immediately taken to the operating room for complete shunt externalization and placement of an external ventricular drain (EVD). He was started on broad-spectrum antibiotics (vancomycin, cefepime and metronidazole) and underwent percutaneous drainage of the pseudocyst in his abdomen. Cultures from both the percutaneous drainage and CSF were positive for acid-fast bacilli (AFB) and presumed NTM. His antibiotics were switched to azithromycin, amikacin and cefoxitin by postadmission day 4 . He continued to decompensate requiring multiple EVD replacements due to loculated ventriculitis. AFB was identified as Mycobacterium abscesses. Intrathecal (IT) amikacin was started on postadmission day 9. This was delayed due to the inability to clamp the EVD and safely administer IT amikacin. During the course of treatment, he had significant QTc interval prolongation which necessitated holding IV azithromycin and starting linezolid with readministration of azithromycin via nasogastric tube to minimize the risk of QTc prolongation by avoiding high peaks. He remained critically ill on a ventilator and required 12 EVD replacements in attempts to clear his loculated hydrocephalus. A brain MRI with contrast showed severe ventriculitis and cerebral inflammation (Figure 2).

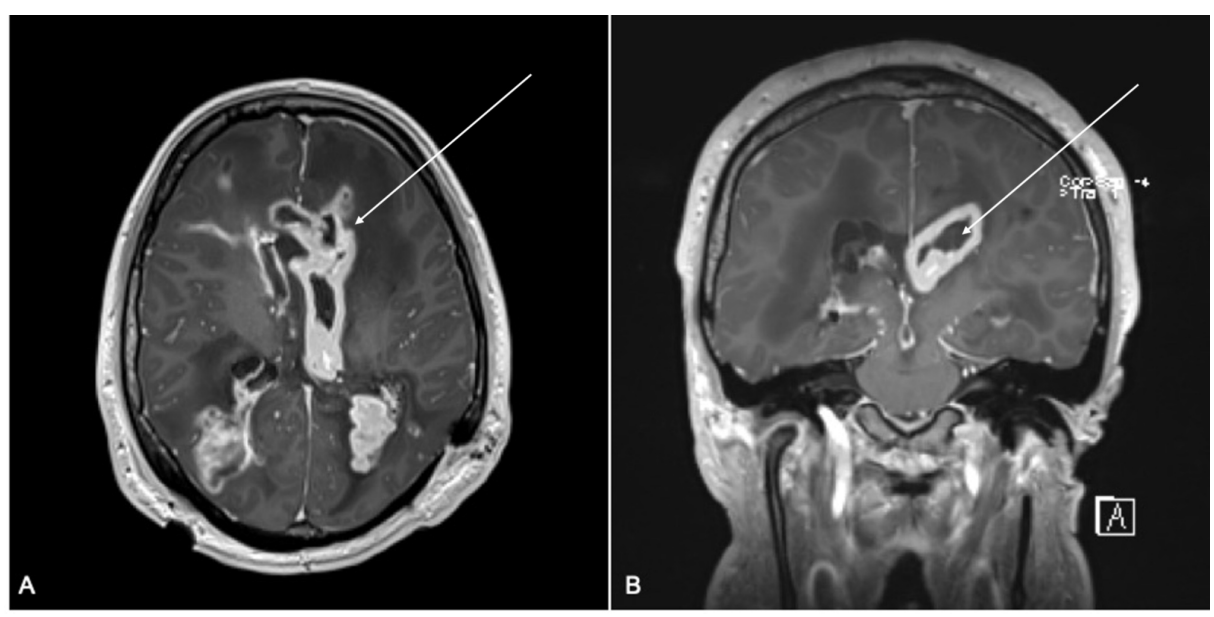

\section{FIGURE 2: MRI T1 with contrast}

MRI T1 with contrast showing severe ventriculitis (arrow) and adjacent cerebral inflammatory changes with edema in axial $(A)$ and coronal $(B)$ sequences.

On postadmission day 30, he was transitioned to comfort care. He passed away on postadmission day 36.

\section{Patient no. 2}

A 71-year-old woman with normal pressure hydrocephalus status post placement of VP shunt approximately two years prior presented with recurrent and prolonged abdominal discomfort. She had a history of multiple abdominal surgeries including gastric lap band complicated by gastric perforation as well as cholecystectomy a year after her shunt was initially placed.

\section{Patient Course}

She was initially admitted with cellulitis and an abscess around an epigastric port site which crossed the VP shunt catheter. She underwent irrigation and drainage of the abscess as well as externalization of the VP shunt at her clavicle. Her hardware was removed completely, and she underwent an endoscopic third ventriculostomy. Abdominal wound catheter site culture grew Mycobacterium fortuitum. CSF remained negative on serial testing. She was treated with imipenem and doxycycline for five weeks, and then switched to oral azithromycin and doxycycline which was continued for three months. Doxycycline was changed to oral sulfamethoxazole-trimethoprim with azithromycin for another 1.5 months. She discontinued antibiotics at approximately six months due to nausea and intolerance. She continued to do well without any sign of infection.

\section{Discussion}


NTM is a rare cause of infection in patients with neurosurgical hardware. We present two cases of serious hardware infection with NTM. These types of infections are rare, with only 19 cases reported (Table 1) [318]. Most cases involved infections with subgroups within the Mycobacterium fortuitum (48\% of cases) and Mycobacterium abscessus ( $43 \%$ of cases). None of the patients were immunocompromised. The age range was 2 to 71 years, with a median age of 45 years. The majority of neurosurgical hardware involved was shunt catheters (70\% of cases). Interestingly, $43 \%$ (9/21) of patients in the series and $64 \%(9 / 14)$ of patients with shunt hardware had symptoms of distal infection with peritonitis, pseudocyst or abscess formation. The majority of patients $(86 \% ; 18 / 21)$ had a positive CSF culture. The majority of device-related NTM infections are caused by Mycobacterium abscessus and Mycobacterium fortuitum, such as in our patients, whereas disseminated disease most commonly caused by Mycobacterium avium complex. Even with rapid hardware explantation and high-dose antibiotic therapy, mortality was 19\% (4/21).

\begin{tabular}{|c|c|c|c|c|c|c|}
\hline Author/year & Age/sex & Organism & Hardware & $\begin{array}{l}+ \\
\text { CSF }\end{array}$ & Treatment & Prognosis \\
\hline \multirow[t]{2}{*}{ Current study } & $25 \mathrm{M}$ & $\begin{array}{l}\text { Mycobacterium } \\
\text { abscessus }\end{array}$ & $\begin{array}{l}\text { VP shunt, } \\
\text { pseudocyst }\end{array}$ & Yes & Azithromycin, amikacin, cefoxitin, IT amikacin & $\begin{array}{l}\text { Death, } 1 \\
\text { month }\end{array}$ \\
\hline & $71 \mathrm{~F}$ & $\begin{array}{l}\text { Mycobacterium } \\
\text { fortuitum }\end{array}$ & $\begin{array}{l}\text { VP shunt, } \\
\text { pseudocyst }\end{array}$ & No & $\begin{array}{l}\text { Imipenem, doxycycline (5 weeks), azithromycin, } \\
\text { bactrim }\end{array}$ & $\begin{array}{l}\text { Stable, } 8 \\
\text { months } \\
\text { f/u }\end{array}$ \\
\hline $\begin{array}{l}\text { Zakrzewski et } \\
\text { al., } 2019 \text { [3] }\end{array}$ & $26 \mathrm{~F}$ & $\begin{array}{l}\text { Mycobacterium } \\
\text { fortuitum }\end{array}$ & LP shunt & Yes & Amikacin, imipenem, moxifloxacin, TMP/SMX & $\begin{array}{l}\text { Stable, } 1 \\
\text { year f/u }\end{array}$ \\
\hline $\begin{array}{l}\text { Xess et al., } \\
2019 \text { [4] }\end{array}$ & $14 \mathrm{~F}$ & $\begin{array}{l}\text { Mycobacterium } \\
\text { fortuitum }\end{array}$ & $\begin{array}{l}\text { VP shunt, } \\
\text { pseudocyst }\end{array}$ & Yes & Linezolid, ofloxacin, clofazimine, clarithromycin & $\begin{array}{l}\text { Stable, } 3 \\
\text { months }\end{array}$ \\
\hline $\begin{array}{l}\text { Giovannenze et } \\
\text { al., } 2018 \text { [5] }\end{array}$ & $46 \mathrm{M}$ & $\begin{array}{l}\text { Mycobacterium } \\
\text { abscessus }\end{array}$ & PEEK implant & No & Amikacin, imipenem, linezolid (6 weeks) & $\begin{array}{l}\text { Stable } 12 \\
\text { months } \\
\text { f/u }\end{array}$ \\
\hline $\begin{array}{l}\text { Moritz et al., } \\
2017[6]\end{array}$ & $62 \mathrm{M}$ & $\begin{array}{l}\text { Mycobacterium } \\
\text { goodii }\end{array}$ & DBS electrode & No & TMP/SMX, doxycycline (6 months) & $\begin{array}{l}\text { Stable, } 12 \\
\text { months } \\
\text { f/u }\end{array}$ \\
\hline $\begin{array}{l}\text { Baidya et al., } \\
2016 \text { [7] }\end{array}$ & $59 \mathrm{M}$ & $\begin{array}{l}\text { Mycobacterium } \\
\text { abscessus }\end{array}$ & VP shunt & Yes & Amikacin, clarithromycin, meropenem & $\begin{array}{l}\text { Death, } 1 \\
\text { month }\end{array}$ \\
\hline $\begin{array}{l}\text { Levy et al., } \\
2016[8]\end{array}$ & $67 \mathrm{~F}$ & $\begin{array}{l}\text { Mycobacterium } \\
\text { abscessus }\end{array}$ & $\begin{array}{l}\text { VP shunt, distal } \\
\text { tubing in } \\
\text { abdomen }\end{array}$ & Yes & Meropenem, amikacin, azithromycin & $\begin{array}{l}\text { Death, } 1 \\
\text { month }\end{array}$ \\
\hline $\begin{array}{l}\text { Montero et al., } \\
2015 \text { [9] }\end{array}$ & $30 \mathrm{M}$ & $\begin{array}{l}\text { Mycobacterium } \\
\text { abscessus }\end{array}$ & $\begin{array}{l}\text { VP shunt, signs } \\
\text { of peritonitis }\end{array}$ & Yes & Amikacin, imipenem, azithromycin, IT amikacin & $\begin{array}{l}\text { Stable, } 4 \\
\text { years } f / u\end{array}$ \\
\hline $\begin{array}{l}\text { Cadena et al., } \\
2014[10]\end{array}$ & $14 \mathrm{M}$ & $\begin{array}{l}\text { Mycobacterium } \\
\text { fortuitum }\end{array}$ & $\begin{array}{l}\text { VP shunt, abd } \\
\text { pseudocyst }\end{array}$ & Yes & $\begin{array}{l}\text { Meropenem, TMP-SMX, moxifloxacin (9 } \\
\text { months) }\end{array}$ & $\begin{array}{l}\text { Stable, } 2 \\
\text { years f/u }\end{array}$ \\
\hline \multirow[t]{3}{*}{$\begin{array}{l}\text { Lee et al., } 2012 \\
\text { [11] }\end{array}$} & $49 \mathrm{M}$ & $\begin{array}{l}\text { Mycobacterium } \\
\text { abscessus }\end{array}$ & Ommaya & Yes & $\begin{array}{l}\text { Amikacin, clarithromycin, imipenem, } \\
\text { minocycline, minofloxacin }\end{array}$ & $\begin{array}{l}\text { Stable, } 1 \\
\text { year f/u }\end{array}$ \\
\hline & $19 \mathrm{~F}$ & $\begin{array}{l}\text { Mycobacterium } \\
\text { abscessus }\end{array}$ & EVD & Yes & $\begin{array}{l}\text { Amikacin, clarithromycin, imipenem, } \\
\text { levofloxacin, moxifloxacin }\end{array}$ & $\begin{array}{l}\text { Stable, } 1 \\
\text { year f/u }\end{array}$ \\
\hline & $28 \mathrm{~F}$ & $\begin{array}{l}\text { Mycobacterium } \\
\text { abscessus }\end{array}$ & Ommaya & Yes & $\begin{array}{l}\text { Amikacin, cefoxitin, clarithromycin, linezolid, } \\
\text { meropenem, moxifloxacin, tigecycline, } \\
\text { TMP/SMX }\end{array}$ & $\begin{array}{l}\text { Stable, } 2 \\
\text { years } \mathrm{f} / \mathrm{u}\end{array}$ \\
\hline $\begin{array}{l}\text { Aliabadi et al., } \\
2008 \text { [12] }\end{array}$ & $60 \mathrm{M}$ & $\begin{array}{l}\text { Mycobacterium } \\
\text { fortuitum }\end{array}$ & Baclofen pump & Yes & $\begin{array}{l}\text { Amikacin, ciptro, clarithromycin, TMP/SMX (19 } \\
\text { months) }\end{array}$ & \\
\hline $\begin{array}{l}\text { Uche et al., } \\
2008 \text { [13] }\end{array}$ & $60 \mathrm{~F}$ & $\begin{array}{l}\text { Mycobacterium } \\
\text { goodii }\end{array}$ & VP shunt & Yes & Imipenem, moxifloxacin & $\begin{array}{l}\text { Stable, } 3 \\
\text { months }\end{array}$ \\
\hline $\begin{array}{l}\text { Viswanathan et } \\
\text { al., } 2004 \text { [14] }\end{array}$ & $60 \mathrm{~F}$ & $\begin{array}{l}\text { Mycobacterium } \\
\text { fortuitum }\end{array}$ & $\begin{array}{l}\text { VP shunt, } \\
\text { abdominal } \\
\text { abscess }\end{array}$ & Yes & Kanamycin, ciprofloxacin & $\begin{array}{l}\text { Stable, } 6 \\
\text { months }\end{array}$ \\
\hline $\begin{array}{l}\text { Quinn et al., } \\
2002[15]\end{array}$ & $12 \mathrm{M}$ & $\begin{array}{l}\text { Mycobacterium } \\
\text { fortuitum }\end{array}$ & $\begin{array}{l}\text { VP shunt, } \\
\text { peritonitis }\end{array}$ & Yes & Amikacin, cefoxitin, clarithromycin, TMP/SMX & $\begin{array}{l}\text { Stable, } 14 \\
\text { months }\end{array}$ \\
\hline
\end{tabular}


10.1093/cid/ciw861

3. Zakrzewski J, Hu K, Neisewander BL, et al.: Mycobacterium fortuitum meningitis: approach to lumboperitoneal shunt infection. South Med J. 2019, 112:217-221. 10.14423/SMJ.0000000000000955

4. Xess AB, Bala K, Panigrahy A, Singh U: Mycobacterium fortuitum as a cause of acute CNS infection in an immune-competent girl undergoing repeated VP shunt surgeries. BMJ Case Rep. 2019, 12:e226900. 10.1136/bcr-2018-226900

5. Giovannenze F, Stifano V, Scoppettuolo G, et al.: Incidental intraoperative diagnosis of Mycobacterium abscessus meningeal infection: a case report and review of the literature. Infection. 2018, 46:591-597. 10.1007/s15010-018-1141-5

6. Moritz DC, Harrington AT, Slavin K, Gomez C, Jarrett OD: Deep brain stimulator infection by a novel rapid growing Mycobacterium. Acta Neurochir. 2017, 159:2239-2241. 10.1007/s00701-017-3335-5

7. Baidya A, Tripathi M, Pandey P, Singh UB: Mycobacterium abscessus as a cause of chronic meningitis: a rare clinical entity. Am J Med Sci. 2016, 351:437-439. 10.1016/j.amjms.2016.02.009

8. Levy ZD, Du V, Chiluwal A, Chalif DJ, Ledoux DE: Ventriculoperitoneal shunt infection with Mycobacterium abscessus: a rare cause of ventriculitis. World Neurosurg. 2016, 86:510-511. 10.1016/j.wneu.2015.10.068

9. Montero JA, Alrabaa SF, Wills TS: Mycobacterium abscessus ventriculoperitoneal shunt infection and review of the literature. Infection. 2016, 44:251-253. https://doi.org/10.1007/s15010-015-0817-3

10. Cadena G, Wiedeman J, Boggan JE: Ventriculoperitoneal shunt infection with Mycobacterium fortuitum: a rare offending organism. J Neurosurg Pediatr. 2014, 14:704-707. https://doi.org/10.3171/2014.8.PEDS13677

11. Lee MR, Sheng WH, Hung CC, Yu CJ, Lee LN, Hsueh PR: Mycobacterium abscessus complex infections in humans. Emerg Infect Dis. 2015, 21:1638-1646. 10.3201/2109.141634

12. Aliabadi H, Osenbach RK: Intrathecal drug delivery device infection and meningitis due to Mycobacterium fortuitum: a case report. Neuromodulation. 2008, 11:311-314. 10.1111/j.1525-1403.2008.00181.x

13. Uche CS, Silibovsky R, Jungkind D, Measley R: Ventriculoperitoneal shunt-associated Mycobacterium goodii infection. Infect Dis Clin Pract. 2008, 16:129-130. 10.1097/IPC.0b013e318142cbae

14. Viswanathan R, Bhagwati SN, Iyer V, Newalkar P: Ventriculo-peritoneal shunt infection by Mycobacterium fortuitum in an adult. Neurol India. 2004, 52:393-394.

15. Quinn JV, Steele RW: Ventriculitis following a neurosurgic procedure. Clin Pediatr (Phila). 2002, 41:439441. 10.1177/000992280204100610

16. Midani S, Rathore MH: Mycobacterium fortuitum infection of ventriculoperitoneal shunt. South Med J. 1999, 92:705-707. 10.1097/00007611-199907000-00009

17. Madaras-Kelly KJ, DeMasters TA, Stevens DL: Mycobacterium fortuitum meningitis associated with an epidural catheter: case report and a review of the literature. Pharmacotherapy. 1999, 19:661-666.

18. Flor A, Capdevila JA, Martin N, Gavalda J, Pahissa A: Nontuberculous mycobacterial meningitis: report of two cases and review. Clin Infect Dis. 1996, 23:1266-1273. 10.1093/clinids/23.6.1266

19. Chan KH, Mann KS, Seto WH: Infection of a shunt by Mycobacterium fortuitum: case report . Neurosurgery. 1991, 29:472-474. 10.1097/00006123-199109000-00028

20. Griffith DE, Aksamit T, Brown-Elliott BA, et al.: An official ATS/IDSA statement: diagnosis, treatment, and prevention of nontuberculous mycobacterial diseases. Am J Respir Crit Care Med. 2007, 175:367-416. 10.1164/rccm.200604-571ST 